\title{
Superoxide dismutase analog (TEMPOL: 4-hydroxy-2, 2, 6, 6- tetramethylpiperidine 1-oxyl) treatment restores erectile function in diabetes-induced impotence
}

\author{
Toshifumi Kawakami, M.D. ${ }^{1,2}$, Shinji Urakami, M.D., Ph.D. ${ }^{1}$, Hiroshi Hirata, M.D., Ph.D. ${ }^{1}$, \\ Yuichiro Tanaka, Ph.D. ${ }^{1}$, Koichi Nakajima, M.D., Ph.D. ${ }^{2}$, Hideki Enokida, M.D., Ph.D. ${ }^{1}$, \\ Hiroaki Shiina, M.D., Ph.D. ${ }^{1}$, Tatsuya Ogishima, M.D. ${ }^{1}$, Takashi Tokizane, M.D., Ph.D. ${ }^{1}$, Ken \\ Kawamoto, M.D., Ph.D. ${ }^{1}$, Kazukiyo Miura, M.D., Ph.D. ${ }^{2}$, Nobuhisa Ishii, M.D., Ph.D. ${ }^{2}$, and \\ Rajvir Dahiya, Ph.D. ${ }^{1,{ }^{* *}}$ \\ ${ }^{1}$ Department of Urology, San Francisco Veterans Affairs Medical Center and University of \\ California at San Francisco, San Francisco, California \\ ${ }^{2}$ Department of Urology, Toho University Faculty of Medicine, Tokyo, Japan
}

\section{Abstract}

We hypothesized that administration of the superoxide dismutase (SOD) mimetic Tempol (4hydroxy-2, 2, 6, 6-tetramethylpiperidine 1-oxyl) may reverse diabetes induced ED(erectile dysfunction). To test this hypothesis, ROS related genes (SOD1, SOD2, GPx1, CAT, NOS2, NOS3), erectile functional studies, and immunohistochemical analysis were performed in diabetic rats treated with or without Tempol. Thirty Sprague-Dawley (3-4 months old) rats were divided into 3 groups ( $\mathrm{n}=10$ each), 20 with diabetes (diabetic control and Tempol treatment) and 10 healthy controls. Twelve weeks after induction of diabetes by streptozotocin and Tempol treatment, all groups underwent in vivo cavernous nerve stimulation. Rat crura were harvested and expression of antioxidative defense enzymes examined by semi-quantitative RT-PCR. To confirm the RT-PCR results, we performed immunohistochemistry (IHC) for catalase (CAT) and iNOS (NOS2). Nitration of tyrosine groups in proteins was also examined by IHC. Mean intracavernous pressure in the diabetic group was significantly lower than in healthy controls $(\mathrm{p}<0.001)$ and was reversed by Tempol treatment ( $\mathrm{p}<0.0108)$. NOS2 protein expression was significantly increased in diabetic animals compared to healthy controls and Tempol restored NOS2 protein level. Nitrotyrosine was also higher in diabetic animals and though Tempol treatment decreased its formation, it remained higher than that found in healthy controls. This study suggests that Tempol treatment increased erectile function through modulating oxidative stress related genes in diabetic rats. This is the first report about the relationship between diabetes induced erectile dysfunction

Users may view, print, copy, and download text and data-mine the content in such documents, for the purposes of academic research, subject always to the full Conditions of use:http://www.nature.com/authors/editorial_policies/license.html\#terms

${ }^{* *}$ To whom correspondence should be addressed: Rajvir Dahiya, Ph.D., Professor and Director, Urology Research Center (112F), Veterans Affairs Medical Center and University of California at San Francisco, 4150 Clement Street, San Francisco, CA 94121,

Phone: 415-750-6964; Fax: 415-750-6639; rdahiya@urology.ucsf.edu.

Conflict of interest

None declared. 
and oxidative stress, and anti-oxidative therapy using the superoxide dismutase mimetic, Tempol to restore erectile function.

\section{Keywords}

oxidative stress; nitric oxide; superoxide; diabetes; erectile dysfunction

\section{INTRODUCTION}

There are 23.6 million children and adults in the United States, or $7.8 \%$ of the population, who have diabetes. While an estimated 17.9 million have been diagnosed with diabetes, 5.7 million people (or nearly one quarter) are unaware that they have the disease. ${ }^{1}$ It has been estimated that $50 \%$ to $75 \%$ of males with diabetes have erectile dysfunction (ED). ${ }^{2}$ In men, $\mathrm{ED}$ is one of the major complications of diabetes.

The penis is a richly vascularized organ, and penile erection is, in large part, a vascular event. Recent reports show that in men with diabetes mellitus, erectile dysfunction is an early event and a potential marker for cardiovascular disease. ${ }^{3}$ Data from several studies involving diabetic patients with cardiac disease have shown a high prevalence (42\% to $75 \%$ ) of ED in the patient population. Also a recent report showed incidence rates of occult cardiovascular disease in men with ED as high as $15 \%$ to $30 \%{ }^{4}$ Therefore ED is considered to be an early manifestation of systemic endothelial dysfunction.

The cause of diabetes-induced ED is multifactorial and its mechanisms are not fully understood. The first line treatment, phosphodiesterase type 5 inhibitor (e.g., Sildenafil, Vardenafil and Tadalafil) is widely used as a medicine to maintain an erection, but in diabetes its effect is clearly reduced when compared with non-diabetic men. ${ }^{5}$ Oxidative stress occurs when reactive oxygen and nitrogen species (ROS and RNS, respectively) production is excessive and/or defense mechanisms fail. ROS are kept under control by an endogenous system of protection that consists of scavenger enzymes (superoxide dismutase, catalase, glutathione peroxidase) and antioxidants (vitamin E, beta-carotene, ascorbic acid, glutathione).

A number of sources of ROS, in particular superoxide anion, have been identified in diabetes including non-enzymatic, e.g. mitochondrial respiratory chain, glucose autoxidation, activation of polyol pathways, glycation-formation of AGEs (advanced glycation end products) and enzymatic, e.g. $\mathrm{NAD}(\mathrm{P}) \mathrm{H}$ oxidase, uncoupled eNOS, xanthine oxydase, cyclooxygenase. ${ }^{6}$ It has been shown that oxidative damage due to hyperglycemia contributes to both macro and microvascular pathology in diabetes such as atherosclerosis, cardiovascular diseases, retinopathy, nephropathy and peripheral neuropathy. ${ }^{7}$ A recent publication has suggested that there is a close relationship between oxidative stress and diabetic ED in both animal models and humans. ${ }^{8}$

Natural anti-oxidants, in particular vitamin E, have demonstrated little beneficial effect in clinical trials. ${ }^{9}$ It has recently been suggested that antioxidant therapy with vitamin $\mathrm{E}$ or 
others is limited to scavenging already formed oxidants, particularly lipid peroxidation products. ${ }^{10}$

Tempol (4-hydroxy-2, 2, 6, 6-tetramethylpiperidine 1-oxyl), is a low molecular weight, water-soluble, membrane permeable and metal-independent superoxide dismutase (SOD) mimetic, which has been widely employed in electron spin resonance spectroscopy. ${ }^{11}$ Tempol can penetrate cell membranes and react with both intra- and extra-cellular oxygen free radicals, resulting in protecting endothelial function. ${ }^{12}$

This property makes Tempol attractive for treatment of diseases related to oxidative stress. Recent studies have indicated that Tempol can be used as an antioxidant in animal models to prevent many diseases that are caused by ROS such as ischemic-reperfusion injury, inflammation and shock. ${ }^{13}$

However in most of these experiments, Tempol has been administered before or at the time of onset of disease. Diabetes mellitus, a continuous hyperglycemia, is a chronic disease, therefore its complications are already established at the time of diagnosis. Our aim in this study was to assess whether delayed and chronic administration of an anti-oxidant superoxide scavenger such as Tempol would be effective as an intervention to diabetic ED complications. To our knowledge there have been no reports about Tempol, ROS and its effect on ED.

\section{MATERIAL AND METHODS}

\section{Experimental animals}

Thirty 3 to 4 month-old (440-560mg) male Sprague-Dawley rats (Charles River Laboratories, Wilmington, Massachusetts) were divided into the following 3 groups: (1) healthy controls, (2) diabetes untreated, (3) diabetes antioxidant (Tempol) treated. The diabetic group received two intraperitoneal injections of $40 \mathrm{mg} / \mathrm{kg}$ storeptozotocin (STZ) (Sigma-Aldrich, Inc., St Louis, Missouri) at 1-week intervals to induce diabetes. STZ was dissolved in citrate phosphate buffer $(0.1 \mathrm{M}$ citric acid and $0.2 \mathrm{M}$ disodium phosphate, $\mathrm{pH}$ 7.0). Control rats were injected with an equal volume of buffer only (Fig. 1). The development of diabetes was confirmed by blood glucose levels 1 week after STZ injection. STZ treated rats with a blood glucose level of below $250 \mathrm{mg} / \mathrm{dl}$ were excluded from this study. Body weight and blood glucose levels in serum samples obtained from the tail vein were measured weekly. Tempol treatment was started 6 weeks after the establishment of diabetes. Subcutaneous injections of $100 \mathrm{mg} / \mathrm{kg}$ Tempol (4-hydroxy-2, 2, 6, 6tetramethylpiperidine 1-oxyl) (Sigma-Aldrich, Inc., St Louis, Missouri) were administered twice a day for 6 weeks.

This project was approved by the animal ethics committee (project number; A3476-01).

\section{Functional study}

Erectile function was assessed by measurement of intracavernous pressure after electro stimulation of the cavernous nerve. ${ }^{14,15}$ Briefly, after anesthesia by inhalation of sevoflurane (Baxter Healthcare Corporation, Deerfield, Illinois), rats were placed in a supine 
position and through a low abdominal midline incision the lateral prostatic space was exposed on each side. The major pelvic ganglia, pelvic nerves and cavernous nerves were identified and exposed. The skin overlying the penis was incised and the penile crura were exposed by removing part of ischiocavernous muscle and fascia. A 23 gauge needle filled with $250 \mathrm{U} / \mathrm{ml}$ heparin solution was inserted into the crura and connected to a pressure monitor with polyethylene tubing. The increase in intracavernous pressure was measured and recorded using LabVIEW 5.0 (National Instruments, Austin, Texas) software. Electro stimulation was performed with a delicate stainless steel bipolar hook electrode and monophasic rectangular voltage pulses were delivered using a computer program. The stimulus parameters were $1.5 \mathrm{~mA}, 20 \mathrm{~Hz}$ frequency, pulse width 0.2 millisecond and a duration of 60 seconds. The cavernous nerve was stimulated 5 times at 4-minutes interval on each side. ICP was measured and recorded with Lab View 5.01 software (National Instruments, Austin, Texas). The ICP is defined as the maximal pressure obtained by stimulation minus the basal pressure before stimulation. The latency period is defined as the time between cavernous nerve stimulation and the onset of pressure rise inside the penile crura.

After the functional study was completed a crura was obtained and incubated in RNAlater RNA Stabilization Reagent (QIAGEN Inc., Valencia, California) overnight at $4 \mathrm{C}$ and the next day the samples were frozen in liquid nitrogen and stored at $-80^{\circ} \mathrm{C}$ until processed for mRNA expression analysis. The crura of the other side was placed in a $4 \%$ formaldehyde solution for immunohistochemical studies of cavernous tissue. After the procedure the rats were sacrificed by thoracotomy.

\section{RNA isolation}

Total RNA was isolated using a combination of TRIzol Reagent (Invitrogen, Carlsbad, California) and RNeasy Mini Kit(QIAGEN Inc., Valencia, California), as described previously. ${ }^{16}$ Tissue was homogenized in TRIzol reagent, chloroform was added and centrifugation performed to improve recovery of the aqueous phase of the solution. Ethanol (100\%) was added to an equal volume of the recovered aqueous solution, and the mixture was added to an RNeasy minicolumn. All subsequent steps were performed as described by the supplier (QIAGEN RNeasy Mini hand book Third Edition). The concentration and quality of RNA was measured by spectrophotometric analysis at 260 and $280 \mathrm{~nm}$.

\section{Reverse transcriptase Polymerase chain reaction (RT-PCR)}

Total RNA was reverse transcribed into cDNA with the use of Reverse Transcription System (Promega Corporation, Madison, Wisconsin) using oligo(dT) as primers. Antioxidative enzyme, such as $\mathrm{Cu} / \mathrm{ZnSOD}$ (SOD1), MnSOD (SOD2), Glutathione peroxidase (GPx1) and catalase (CAT), and also nitric oxide synthase, inducible (iNOS; NOS2) and endothelial (eNOS; NOS3) were analyzed by a semi-quantitative method. Primer sets used for RT-PCR are shown in Table 1. PCR cycle parameters consisted of a 1 minute denaturing step at 94C, 1 minute annealing step at 58C, 1 minute extension step at $72 \mathrm{C}$ and 40 cycles were used per amplification. Beta-Actin served as an internal control. The expression of SOD1, SOD2, GPx1, CAT, NOS2 and NOS3 relative to Beta-Actin was quantified by densitometry. 


\section{Immunohistochemistry}

Immunostaining was performed on formalin fixed, paraffin embedded rat crura tissue sections. Five $\mu \mathrm{m}$ sections were deparaffinized with xylene and rehydrated in graded alcohols followed by rinsing with distilled water. Antigen retrieval was carried out by autoclaving the slides in $10 \mathrm{mM}$ sodium citrate buffer ( $\mathrm{pH}$ 6.0). Endogenous peroxidase activity was blocked with $3 \% \mathrm{H} 2 \mathrm{O} 2$. Incubation with goat anti-rabbit serum (Santa Cruz Biotechnology, Santa Cruz, California) was used to block non-specific protein binding. The nitrotyrosine antibody (Rabbit polyclonal IgG, cat\# 06-284, Upstate Cell Signaling solutions, Lake Placid, New York) was diluted to $2.5 \mu \mathrm{g} / \mathrm{ml}$ and incubated with slides overnight. To confirm the RT-PCR results, we performed IHC for catalase (CAT) (\#ab52477, Abcam, Cambridge, MA) and iNOS (NOS2) (\#53584, Anaspec, San Jose, CA). Omitting the primary antibody was used as negative control. Slides were then incubated with biotinylated secondary antibody to IgG (Santa Cruz Biotechnology, Santa Cruz, California). After washing, the slides were next incubated with HRP-streptavidin (Santa Cruz Biotechnology, Santa Cruz, California), washed and stained with diaminobenzidine (LAB Vision Corporation, Fremont, California) and counterstained with hematoxylin.

\section{Statistics}

All data are expressed as the mean $\pm \mathrm{SE}$ and were analyzed using ANOVA with Bonferroni/ Dunn post hoc test for multiple group comparisons on Statview 5. 0. 1 (SAS Institute, Cary, North Carolina).

\section{RESULTS}

\section{Development of diabetes}

At 12 weeks after STZ injections diabetic rats (diabetic control and Tempol treatment) were significantly lighter in body weight than age matched healthy controls $(452.0 \pm 6.6,448.6 \pm$ 21.8 vs $603.6 \pm 11.8 \mathrm{gm}, \mathrm{p}<0.05)$ and had higher levels of blood glucose $(361.8 \pm 18.2$, $368.7 \pm 19.7 \mathrm{vs} 109.4 \pm 4.0 \mathrm{mg} / \mathrm{dl}, \mathrm{p}<0.05)$. They also showed the usual signs of diabetes, such as polydipsia and polyuria. In diabetic rats, Tempol injections did not have a significant impact on body weight or on blood glucose levels.

\section{Erectile function}

Fig. 1 shows erectile function in diabetic, healthy control and Tempol treated rats. Maximam intracavernous pressure in diabetes was generally lower compared to that observed in healthy controls ( $78.8 \pm 5.6$ vs $130.5 \pm 4.8 \mathrm{~cm} \mathrm{H}_{2} \mathrm{O}, \mathrm{p}<0.05$ ). In addition the pressure was not maintained and declined even further with time. Tempol treatment partially restored intracavernous pressure in diabetic ED $(101.7 \pm 5.6$ vs $78.8 \pm 5.6 \mathrm{~cm} \mathrm{H} 2 \mathrm{O}, \mathrm{p}<0.05)$. Tempol treatment reversed about $44.3 \%$ of the diabetes-induced reduction in intracavernous pressure.

\section{RT-PCR analysis}

Figure 2 shows the relative levels of SOD1, SOD2, GPx1, CAT, NOS2 and NOS3 mRNA transcripts in the penile rat crura. SOD2 mRNA expression was significantly lower in 
diabetic rats compared to healthy controls (Fig 3-B). Tempol treated diabetic rats had increased mean levels of SOD2 mRNA expression. CAT mRNA expression in diabetic rats was significantly lower compared to healthy controls (Fig 3-D). Tempol treated animals had CAT mRNA levels similar to those in healthy controls. NOS2 (iNOS) mRNA expression in diabetic rats was significantly higher compared to healthy controls (Fig 3-E). Tempol treated rats had NOS2 mRNA levels lower than that of healthy controls.

SOD1, GPx1 and NOS3 mRNA expression did not significantly change among diabetic animals, healthy controls and the Tempol treated group (Fig 3-A, C, F).

\section{Immunohistochemistry of rat crura}

To confirm the RT-PCR results, we performed IHC for catalase (CAT) and NOS2 (iNOS). We did not find a significant difference in CAT expression between DM and the Tempol treatment group (data not shown). However NOS2 protein levels were dramatically decreased in the Tempol treated group and this result was similar to the RT-PCR results. Typical NOS2 (iNOS) immunostaining is shown in Fig. 4.

NOS2 (iNOS) was stained in penile cavernosal smooth muscle and endothelium. Fig. 5 shows that nitrotyrosine protein expression in the artery wall and smooth muscle cells was higher in diabetic rats than in healthy controls. Tempol treatment decreased nitrotyrosine formation but not to healthy control levels.

\section{Discussion}

This is the first report to demonstrate the relationship between diabetes induced erectile dysfunction, oxidative stress, and anti-oxidative therapy using the superoxide dismutase mimetic, Tempol, to restore erectile function. Diabetic erectile dysfunction invilves several factors including NO, ROS, NOS (NOS1, NOS2, and NOS3), peroxynitrite, and others. Nitric oxide (NO), a vasodilator, is a principal mediator in the initiation and maintenance of penile erection. NO production plays a central role in modulating endothelial function. ${ }^{17}$ Decreased relaxation of the cavernosal tissue in experimental diabetic animals is associated with a lack of NO bioavailability. ${ }^{18}$ Vascular endothelial cells are an important target of hyperglycemic damage. It has been shown that hyperglycemia increases the production of ROS inside cultured bovine aortic endothelial cells. ${ }^{19} \mathrm{~A}$ recent study demonstrated that the hyperglycemia induced process of overproduction of superoxide at the mitochondrial level, is the initial and key event in the activation of all other pathways involved in the pathogenesis of diabetic complications. ${ }^{20}$ Hyperglycemia also favors increased expression of iNOS (NOS2) through the activation of stress sensitive pathways such as NFkB which can increase the generation of NO. ${ }^{21}$ Superoxide immediately interacts with NO, generating cytotoxic peroxynitrite (ONOO-), thereby reducing the efficacy of the potent endothelium derived vasodilator system that participates in the homeostatic regulation of the vasculature. Peroxynitrite, a potent oxidative stress inducer, leads to the iNOS (NOS2) and eNOS (NOS3) uncoupled state, due to a deficiency of tetrahydrobiopterin (BH4), a NOS cofactor, resulting in the production of superoxide rather than NO. ${ }^{7}$ It has also been shown that peroxynitrite induces nitrotyrosine formation, lipid peroxidation, DNA strand breakage and consequent activation of the nuclear enzyme poly (ADP-ribose) polymerase (PARP). PARP 
activation in turn depletes the intracellular concentration of its substrate NAD+, slowing the rate of glycolysis, electron transport, and ATP formation, and produces the ADPrybosylation of GADPH, resulting in endothelial dysfunction. ${ }^{22}$

Based on this evidence, we examined the mRNA and protein levels of antioxidative enzymes in control, diabetes, and Tempol treated rats to confirm the effect of Tempol on rat crura.

We observed decreased catalase (CAT) mRNA expression in diabetes but there was no change in CAT protein levels between control and diabetes. We did not examine CAT activity however our results may suggest that Tempol does not affect CAT expression. In our study, NOS2 (iNOS) mRNA and protein levels increased in diabetic rats and this result is consistent with a previous report. ${ }^{23}$ Also, the protein expression level of iNOS (NOS2) was restored in Tempol treated rats. We also found higher nitrotyrosine protein expression in diabetic rats in the artery wall and smooth muscle cells with decreased nitrotyrosine protein levels in Tempol treated rats. However it is unknown why Tempol affected peroxynitrite expression levels. Additonal studies are needed to address this. Under potent oxidative stress conditions such as diabetes, peroxynitrite induces NOS2 (iNOS) and nitrotrysine in various tissues. ${ }^{24-26}$ Nitrotyrosine formation mediates apoptosis and pathological angiogenesis in endothelial cells. ${ }^{27-29}$

A previous report from our laboratory suggested that diabetic rats have enhanced apoptotic activity in rat crura. ${ }^{30}$ In this study we did not perform apoptosis analysis in Tempol treated rats crura. However, our results showing that hyperglycemia induced nitrotyrosine formation and NOS2 production suggest that there may be increased apoptosis in diabetic rat crura. Thus Tempol might inhibit apoptosis in diabetic rat crura and restore endothelial function.

In this study Tempol treatment was started at 6 weeks after the onset of diabetes. Our previous results have indicated that low intracavernous pressure was observed 4 weeks after the onset of diabetes. ${ }^{30}$ Since diabetes is a chronic disease, Tempol was administered to rats with established diabetic induced ED. The delayed and continuous Tempol treatment regimen used in this study partially restored erectile function but we hypothesize that if Tempol was administered before or at the onset of diabetes, its effect might be more dramatic.

In conclusion, the administration of Tempol to diabetic rats inhibited superoxide overproduction. It also reversed the increase of iNOS mRNA expression in rat crura and release of nitric oxide from endothelial cells leading to decreased formation of peroxynitrite. In addition, decreased peroxynitrite lead to functional restoration of endothelial cells and in turn erectile function. Suppression of oxidative stress is potentially a new strategy for treating diabetes induced erectile dysfunction.

\section{Acknowledgments}

We thank Dr. Roger Erickson for his support and assistance with the preparation of the manuscript. This study was supported by Grants RO1DK075524, T32DK007790 from the NIH. 


\section{References}

1. American Diabetes Association. [accessed 3 September 2008] http://www.diabetes.org/aboutdiabetes.jsp

2. Hakim LS, Goldstein I. Diabetic sexual dysfunction. Endocrinol Metab Clin North Am. 1996; 25:379-400. [PubMed: 8799705]

3. Billups KL. Erectile dysfunction as an early sign of cardiovascular disease. Int J Impot Res. 2005; 17:19-24. [PubMed: 15510194]

4. Schwarz ER, Rastoji S, Rodriguez JJ, Kapur V, Sulemanjee N, Gupta R. A multidisciplinary approach to assess erectile dysfunction in high-risk cardiovascular patients. Int J Impot Res. 2005; 17:37-43.

5. Rendell MS. Sildenafil for treatment of erectile dysfunction in men with diabetes: a randomized controlled trial. Sildenafil Diabetes Study Group. JAMA. 1999; 281:421-426. [PubMed: 9952201]

6. Johanson JS, Harris AK, Rychly DJ, Ergul A. Oxidative stress and the use of antioxidants in diabetes: Linking basic science to clinical practice. Cardiovasc Diabetol. 2005; 4:5. [PubMed: 15862133]

7. Ceriello A. New insights on oxidative stress and diabetic complications may lead to a "causal" antioxidant therapy. Diabetes Care. 2003; 26:1589-1596. [PubMed: 12716823]

8. Musicki B, Burnett AL. Endothelial dysfunction in diabetic erectile dysfunction. Int J Impot Res. 2007; 19:129-138. [PubMed: 16775612]

9. Marchioli R, Schweiger C, Levantesi G, Tavazzi L, Valagussa F. Antioxidant vitamins and prevention of cardiovascular disease: epidemiological and clinical trial data. Lipids. 2001; 36:S5363. [PubMed: 11837994]

10. Cuzzocrea S, Riley DP, Caputi AP, Salvemini D. Antioxidant therapy: a new pharmacological approach in shock, inflammation, and ischemia/reperfusion injury. Pharmacol Review. 2001; 53:135-159.

11. Muscoli C, Cuzzocrea S, Riley DP, Zweier JL, Thiemermann C, Wang ZQ, et al. On the selectivity of superoxide dismutase mimetics and its importance in pharmacological studies. Br J Pharmacol. 2003; 140:445-460. [PubMed: 14522841]

12. Simonsen U, Rodriguez-Rodriguez R, Dalsgaard T, Buus NH, Stankevicius E. Novel approaches to improving endothelium-dependent nitric oxide-mediated vasodilation. Pharmacol Rep. 2009; 61:105-115. [PubMed: 19307698]

13. Thiemermann C. Membrane permeable radical scavengers (tempol) for shock, ischemia reperfusion injury, and inflammation. Crit Care Med. 2003; 31:76-84.

14. Quinlan DM, Nelson RJ, Partin AW, Mostwin JL, Walsh PC. The rat as a model for the study of penile erection. J Urol. 1989; 141:656-661. [PubMed: 2918611]

15. Yamanaka M, Shirai M, Shiina H, Tanaka Y, Enokida H, Tsujimura A, et al. Vascular endothelial growth factor restores erectile function through nhibition of apoptosis in diabetic rat penile crura. J Urol. 2005; 173:318-323. [PubMed: 15592104]

16. Sullivan CJ, Teal TH, Luttrell IP, Tran KB, Peters MA, Wessells H. Microarray analysis reveals novel gene expression changes associated with erectile dysfunction in diabetic rats. Physiol Genomics. 2005; 23:192-205. [PubMed: 16118269]

17. Moncada S, Palmar RM, Higgs EA. Nitric Oxide:physiology, pathophysiology, and pharmacology. Pharmacol Rev. 1991; 43:109-142. [PubMed: 1852778]

18. Bivalacqua TJ, Usta MF, Champion HC, Adams D, Namara DB, Abdel-Mageed AB, et al. Gene transfer of endothelial nitric oxide synthase partially restores nitric oxide synthesis and erectile function in streptozotocin diabetic rats. J Urol. 2003; 169:1911-1917. [PubMed: 12686872]

19. Giardino I, Edelstein D, Brownlee M. BCL-2 expression or antioxidants prevent hyperglycemiainduced formation of intracellular advanced glycation endproducts in bovine endothelial cells. $\mathrm{J}$ Clin Invest. 1996; 97:1422-1428. [PubMed: 8617874]

20. Brownlee M. Biochemistry and molecular cell biology of diabetic complications. Nature. 2001; 414:813-820. [PubMed: 11742414] 
21. Spitaler MM, Graier WF. Vascular targets of redox signaling in diabetes mellitus. Diabetologia. 2002; 45:476-494. [PubMed: 12032623]

22. Soriano FG, Virag L, Jagtap P, Szabo E, Mabley JG, Liaudet L. Diabetic endothelial dysfunction: the role of poly (ADP-ribose) polymerase activation. Nat Med. 2001; 7:108-113. [PubMed: 11135624]

23. Usta MF, Bivalacqua TJ, Yang DY, Ramanitharan A, Sell DR, Viswanathan A, et al. The protective effect of aminoguanidine on erectile function in streptozotocin diabetic rats. J Urol. 2003; 170:1437-1442. [PubMed: 14501785]

24. Ren XY, Li YN, Qi JS, Niu T. Peroxynitrite-induced protein nitration contributes to liver mitochondrial damage in diabetic rats. J Diabetes Complications. 2008; 22:357-364. [PubMed: 18413185]

25. Cheng X, Xia Z, Leo JM, Pang CC. The effect of N-acetylcysteine on cardiac contractility to dobutamine in rats with streptozotocin-induced diabetes. Eur J Pharmacol. 2005; 519:118-126. [PubMed: 16111676]

26. Rao VS, Santos FA, Silva RM, Teixiera MG. Effects of nitric oxide synthase inhibitors and melatonin on the hyperglycemic response to streptozotocin in rats. Vascul Pharmacol. 2002; 38:127-130. [PubMed: 12402510]

27. Taniyama Y, Griendling KK. Reactive oxygen species in the vasculature: Molecular and cellular mechanisms. Hypertension. 2003; 42:1075-1081. [PubMed: 14581295]

28. Ceriello A, Quagliaro L, D’Amico M, Di Filippo C, Marfella R, Nappo F, et al. Acute hyperglycemia induces nitrotyrosine formation and apoptosis in perfused heart from rat. Diabetes. 2002; 51:1076-1082. [PubMed: 11916928]

29. Grishko V, Rachek L, Musiyenko S, Ledoux SP, Wilson GL. Involvement of mtDNA damage in free fatty acid-induced apoptosis. Free Radic Biol Med. 2005; 38:755-762. [PubMed: 15721986]

30. Yamanaka M, Shirai M, Shiina H, Tanaka Y, Tsujimura A, Matsumiya K, et al. Diabetes induced erectile dysfunction and apoptosis in penile crura are recovered by insulin treatment in rats. J Urol. 2003; 170:291-297. [PubMed: 12796708] 


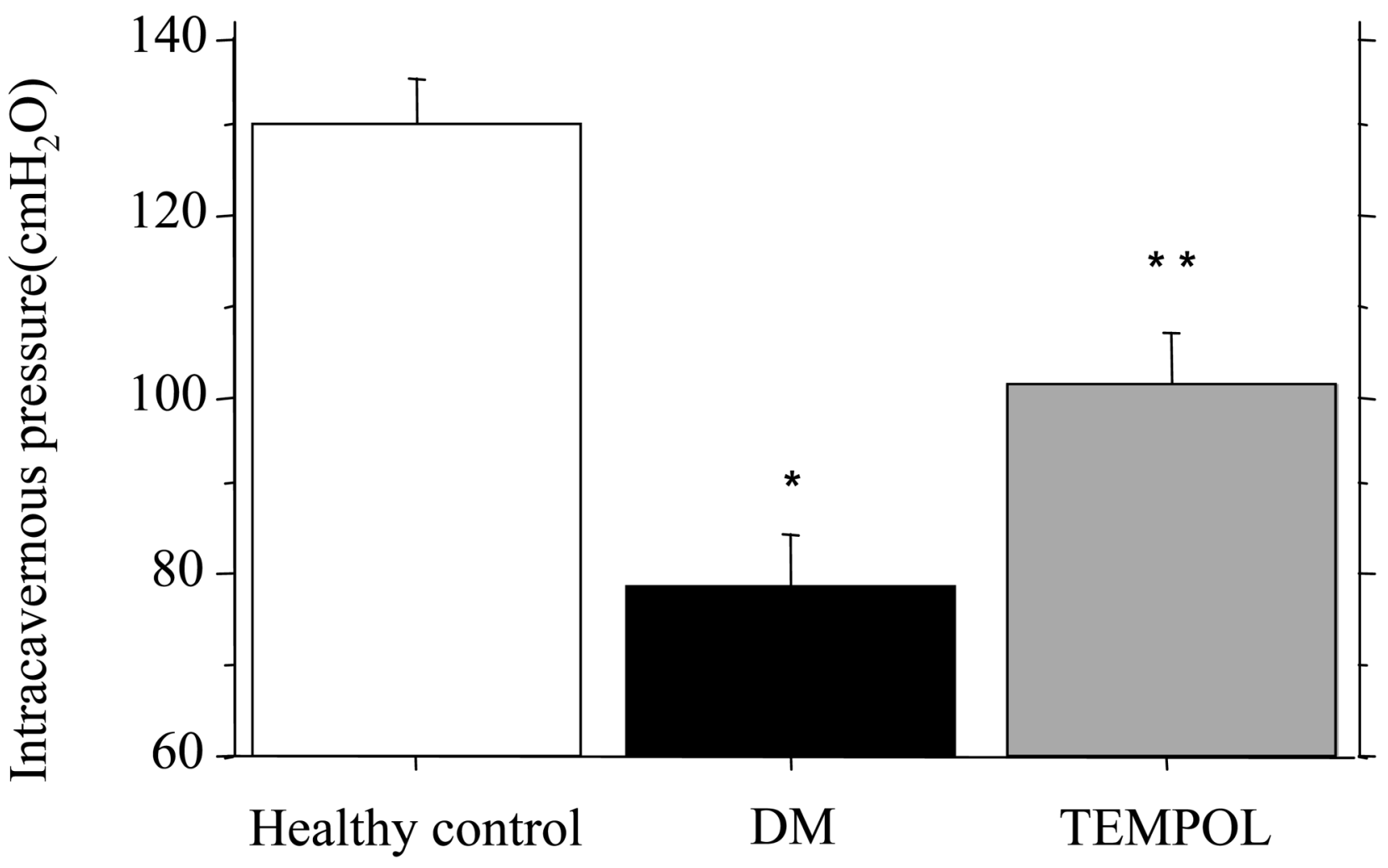

Figure 1.

Intracavernous pressure, Mean maximum pressure in DM was significantly lower than healthy control's. Tempol treatment partially restored in the cavernous pressure.

Note; *Healty control vs DM, $\mathrm{p}<0.0001$; ** DM vs Tempol treatment, $\mathrm{p}<0.01$ 


\section{RT-PCR}

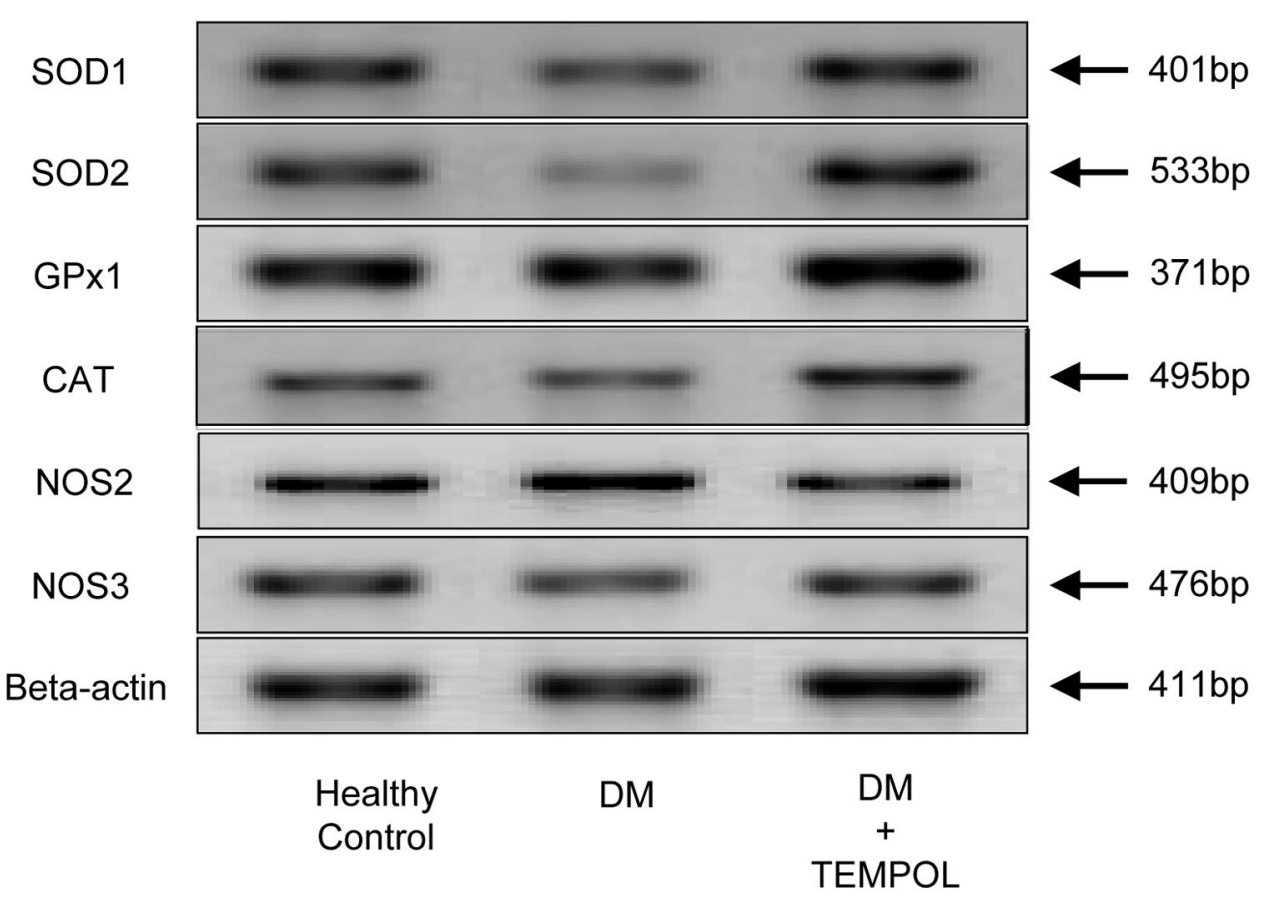

Figure 2.

SOD1, SOD2, GPx1, CAT, NOS2 and NOS3 mRNA expression in penile rat crura from healthy control, diabetic controls and following Tempol treatment. 

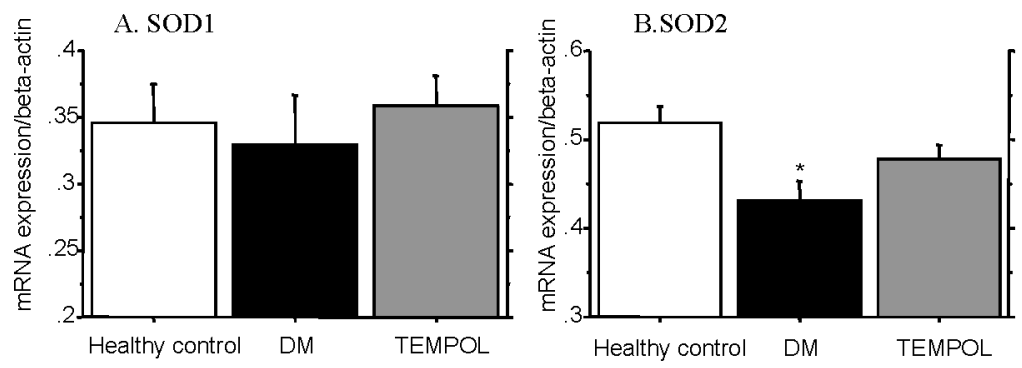

Note; * Healty control vs DM, $p=0.0033$
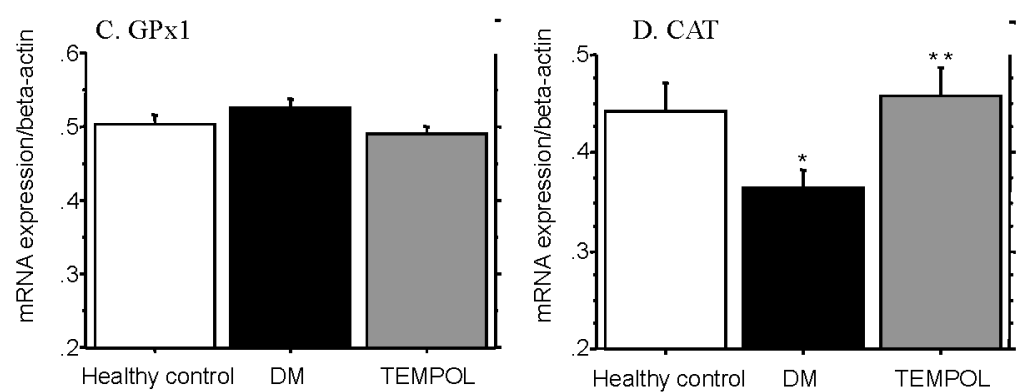

Note; * Healty control vs $D M, p=0.03$ * * DM vs Tempol treatment, $\mathrm{p}=0.01$
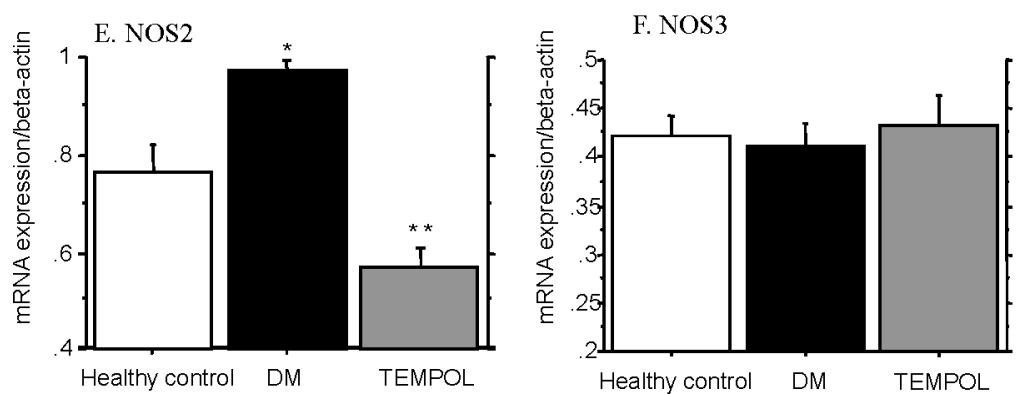

Note; * Healty control vs DM, $p=0.01$

* * DM vs Tempol treatment, $p<0.0001$

Figure 3.

Mean quantitative gene expression of SOD1 (A), SOD2 (B), GPx1 (C), CAT (D), NOS2 (E) and NOS3 (F) was analyzed using densitometry. In SOD1 mRNA expression there was no significant change among healthy controls, DM controls and the Tempol treatment group. However SOD2 mRNA expression in DM was significantly lower than healthy control's and Tempol treatment partially restored its expression but did not reach significance. Glutathione peroxidase (GPX1) mRNA expression (C) was similar in all groups. CAT mRNA expression (D) in DM was significantly lower than in healthy controls and Tempol treatment restored CAT to healthy control level. NOS2 mRNA expression (E) in DM was significantly higher than healthy controls and Tempol treatment mRNA was lower than healthy controls. In NOS3 mRNA expression (F) there was no significant change among diabetes, healthy control and Tempol treatment group. 

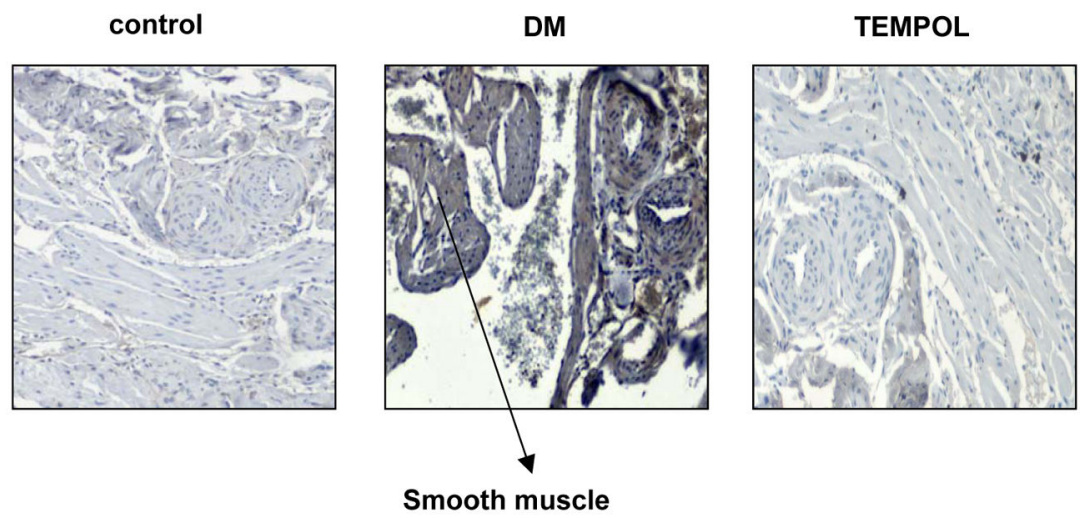

Figure 4.

Representative immunohistochemical staining of rat corpus cavernosum of NOS2 (iNOS). Smooth muscle and endothelium are stained brown in diabetic rats. Magnification is x100. 


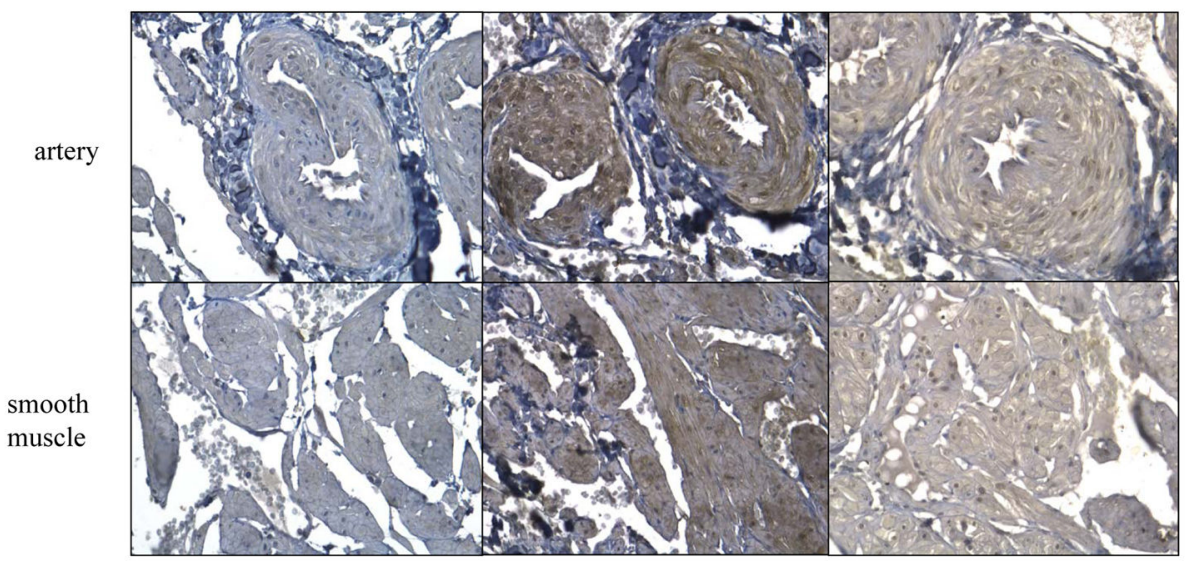

Healthy control

DM

Tempol

Figure 5.

Immunohistochemistry of anti-nitrotyrosine. Nitrotyrosine protein expression in artery wall and smooth muscle cells of rat crura was higher in diabetic rats than in healthy controls. Tempol treatment decreased nitrotyrosine formation. Magnification is x 200 . 


\section{Table 1}

RT-PCR primers

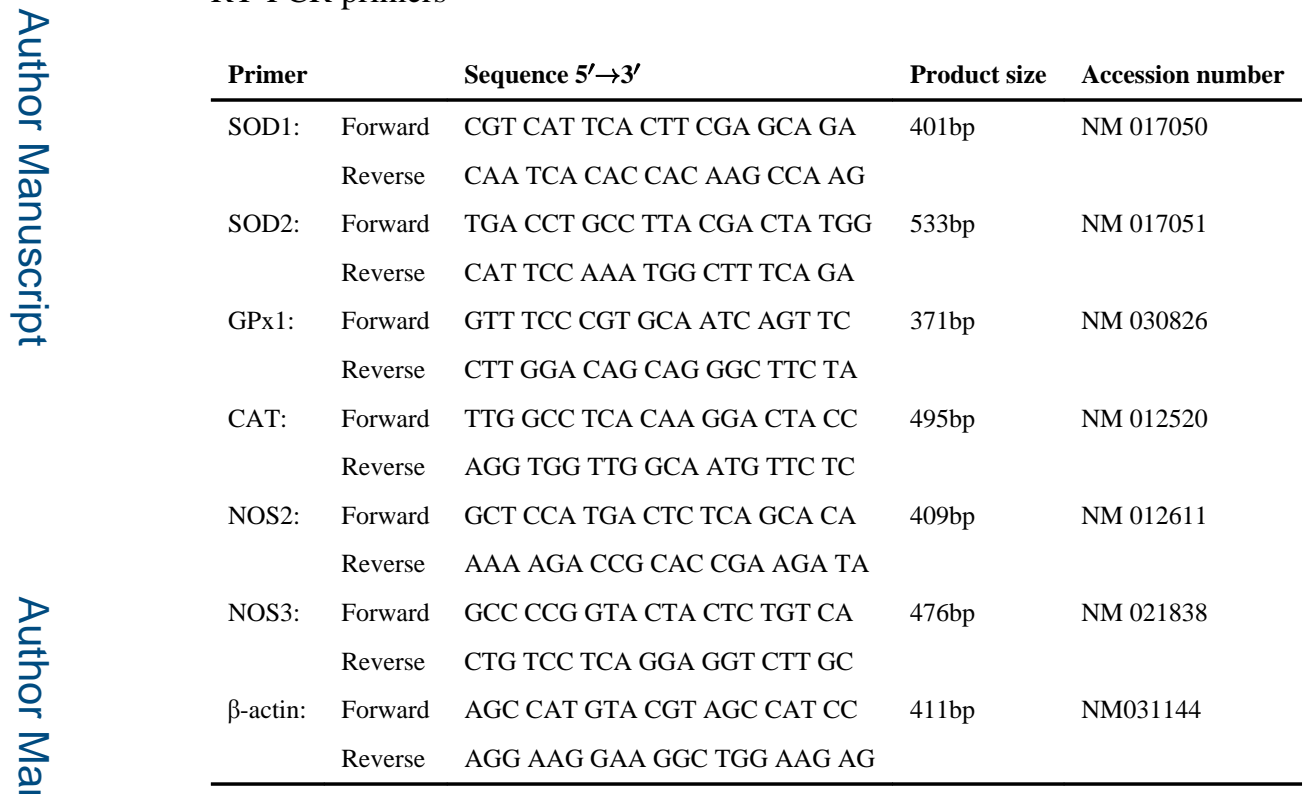

